\title{
Síndrome de Rett. Descripción de dos casos
}

\author{
Rett's síndrome. Report of two cases
}

\author{
CAMPOS Patricia*, MICHELENA María**, BANCALARI Ernesto*** \\ * Profesora Asociada, Departamento de Neurología, Universidad Peruana Cayetano \\ Heredia \\ **Profesora del Departamento de Genética de la Universidad Peruana Cayetano Heredia \\ ***Servicio de Neurología, Hospital Nacional Cayetano Heredia
}

\section{INTRODUCCIÓN}

El Síndrome de Rett (SR) fue descrito inicialmente en 1966 por Andreas Rett. Comprende una involución mental progresiva de inicio antes de los dos años acompañada de pérdida de contacto social y movimientos estereotipados de las manos, apraxia /ataxia de la marcha y crisis de hiperventilación, presentándose exclusivamente en mujeres (1).

Se presenta en todos los grupos étnicos, alcanzando una prevalencia de 1: 10,00012,000 en países europeos, prevalencia que es igual a la de la fenilcetonuria (2).

Presentamos el relato de dos casos vistos dentro de los últimos 7 años, para llamar la atención hacia esta patología que no es frecuente en nuestro medio y que a menudo es erróneamente diagnosticada de autismo infantil.

\section{CASO $\mathrm{N}^{0} 1$}

GSC de 6 años de edad, en la primera consulta en octubre 1986.Relatan los familiares que en una niña supuestamente normal hasta los 3 años, su lenguaje empieza a disminuir, se aísla rechazando el contacto familiar y se muestra agresiva llegando a morderlos; progresivamente va perdiendo las habilidades adquiridas al punto de no valerse por ella misma y volver a usar pañales. Aproximadamente 8 meses después del inicio notan conductas bizarras sobre todo con movimientos repetitivos de las manos, rotaciones sobre el propio eje del cuerpo y comienza a caminar con una marcha cada vez más inestable. Antecedentes de Importancia: No hay consanguineidad de los padres. Embarazo y parto sin complicaciones. Desarrollo psicomotor normal, Examen Clínico: paciente despierta pero aislada del medio, no establece contacto ni siquiera visual con el examinador, rechaza de primera intención el contacto físico, grita frecuentemente y presenta movimientos rotatorios y como de "lavado de manos" constantemente, No presenta evidencia de localización motora, sensitiva o cerebelosa y si apraxia importante en la marcha. En los últimos años surge un franco deterioro de las funciones superiores. Ex. Auxiliares: EEG normal, errores congénitos de metabolismo negativos; Cariotipo normal, Tomografía axial computarizada normal.

\section{CASO $\mathrm{N}^{\circ} 2$}

P.C.M. de 5 años de edad al momento de la primera consulta en diciembre 1991. Natural y procedente de Lima. Según relato de los padres era una niña con un desarrollo 
psicomotor normal hasta los 2 años de edad cuando presentó por primera vez crisis convulsivas tónicas y tónico clónicas generalizadas, paralelamente notaron involución de las funciones superiores y cambios en la marcha, se caía frecuentemente y caminaba como "ebria".

No precisan en que momento notaron aislamiento cada vez mayor del medio y movimientos raros de las manos. Antecedentes: No hay consanguineidad de los padres, nació de parto normal, pero microcefálica. Al examen clínico se encuentra una niña con P.C. de $48 \mathrm{~cm}$; facies no característica (Figura $\mathrm{N}^{\circ} 1$ ) desconectada del medio aunque sin rechazar el contacto de la madre, con movimientos estereotipados de "aplauso" en las manos, crisis de hiperventilación y postura en flexión, no hay déficit motor ni alteración del tono o de los reflejos tendinosos profundos, no hay reflejos anormales y presenta apraxia (Figura $\mathrm{N}^{\circ} 2$ ).

\section{DISCUSION}

El autismo infantil originalmente descrito por Kanner reconoce dentro de su diagnóstico diferencial retardo mental, esquizofrenia infantil, afasia evolutiva y últimamente al SR (II). Sin embargo el paciente con SR se diferencia en presentar ataxia, crisis de hiperventilación, estereotipos motoros simples y repetitivos y movimientos de manos (3); en tanto que el niño autista presenta estereotipos complejos y verbales pero no regresión motora. Las otras características como pobre contacto visual, rechazo pérdida de interés por el medio y problemas de lenguaje hablado no revisten diferencias $(4,5)$.

El desarrollo psicomotor, la historia de embarazo, parto y desarrollo de estas niñas suele ser normal hasta los 12-18 meses de vida $(4,5)$.

Se describen, crisis de gritos sin motivo como la alteración de comportamiento más frecuente y precoz. Nomura et al proponen que ya el desarrollo sería atrasado desde el inicio porque estas niñas suelen inclusive ser hipotónicas.

Criterios diagnósticos de inclusión:

1. Infantes normales desde el nacimiento.

2. Detención del crecimiento del perímetro cefálico entre 3 meses y 4 años.

3. Pérdida de habilidades motoras manuales y/o pérdida de interés en el juego entre 9 meses y 2 años y medio.

4. Regresión psicomotora entre pocos meses y 2 años y medio.

5. Movimientos de las manos "clásicos" y estereotipados aparecidos después del año hasta los 3 años.

6. Apraxia de marcha entre 2 y 4 años.

De acuerdo con Hagberg y Witt-Engerstrom (2) la enfermedad presenta cuatro estadios: Estadio I: Detención del desarrollo, entre 6 a 18 meses de vida, señales inespecíficas de compromiso neurológico.

Estadio II: Rápida regresión psicomotora con cambios de personalidad, comienza alrededor de 1 a 2 años y en muchos pacientes este es el estadio en que pierden el contacto y las habilidades manuales y de lenguaje. 
Estadio III: Aparece cuando la niña ya claramente deficiente mental, parece volver a una personalidad normal, con rasgos autistas menos claros, es un período "pseudoestacionario" que ocurre alrededor de los 3-4 de edad, pero puede ocurrir después e inclusive permanecer en él por muchos años.

Estadío IV: Ocurre ya cerca a la adolescencia y se caracteriza por un franco deterioro neurológico, el que muestra cuadro piramidal, apraxia y ataxia y también pueden ocurrir compromisos de neurona motora inferior. Excepcionalmente predomina el componente distónico haciendo difícil el diagnóstico.

Tres cuartas partes de los paciente con SR presentan epilepsia. En el síndrome clásico ésta empieza en el final del estadío II o en el III, sin embargo suele presentarse mayormente antes de los 10 años. Las crisis pueden ser variadas en su expresión clínica y a menudo son crisis de difícil control, las mismas que sin embargo pueden mejorar espontáneamente al entrar a la adultez (4).

El EEG suele ser normal en los primeros estadíos (6) y sucesivamente aparecen cambios no específicos que comienzan con lentificación difusa, pérdida de las características normales durante sueños y la presencia de actividad paroxismal multifocal seguida de actividad punta-onda lenta difusa. Hagne et al (7), reportan que la secuencia de cambios EEG en pacientes estudiados por ellas comienza con la presencia de puntas rolándicas, las cuales luego se tornan patrones epilépticos de otro tipo y en estadíos avanzados el patrón es delta pseudo periódico y ocasionalmente acompañado de actividad paroxismal periódica generalizada. Han sido descritos patrones semejantes a los de la Panencefalitis esclerosante subaguda en estadió IV.

El diagnóstico clínico no es difícil de hacer si se utilizan los criterios de diagnósticos, sin embargo, la época en que este diagnóstico se hace más difícil es alrededor de los dos años, cuando empieza el período de regresión (8); es precisamente en este período donde el diagnóstico diferencial más importante se hace con el autismo de kanner.

En Europa el diagnóstico más frecuente a ser considerado es la forma infantil de la lipofucsinosis ceroide o enfermedad de Santavouri la cual presenta rápidamente componente mioclónico, degeneración macular, atrofia cortical en la TAC y un EEG con cambios típicos. Deben ser considerados también dentro del diagnóstico diferencial aminoacidopatías y diferentes enfermedades metabólicas, y la forma ataxica de parálisis cerebral. Recientemente debe añadirse un síndrome mas a la lista de diagnóstico diferencial: síndrome de Angelman (happy puppet síndrome)(6,9), el cual reconoce como características ataxia, movimientos estereotipados, conductas autistas y microcefalia adquirida y progresiva, sin embargo no existe la regresión motora encontrada en el SR y se encuentra frecuente y precozmente crisis de risa. Se ha encontrado una anormalidad cromosómica en 5 de 11 niños en el cromosoma 15q.

El diagnóstico diferencial incluye además de lo ya mencionado, encefalopatías tóxicas, encefalopatías epilépticas, síndromes neurocutáneos, formas ataxicas de parálisis cerebral.

Desde el punto de vista bioquímico, genético e histopatológico no se han descrito hallazgos que sean específicos para el SR, parece que existiría una mayor frecuencia de $\mathrm{X}$-frágil pero que no es significativo; de otro lado cuadros disgenéticos o genéticos 
restringidos al sexo femenino y con regresión psicomotora dentro de sus variables, no son muy frecuentes. Lo que actualmente es más aceptado es la teoría de mutación ligada al X-dominante con aborto espontáneo y precoz del feto masculino. Sin embargo el hecho de haber encontrado casos en hermanas sugiere mutación de genes recesivos (1013).

Las características de ambos casos descritos llenan los requisitos aceptados para el Síndrome e inclusive siguen la secuencia de los estadíos, una de ellas presente epilepsia tipo síndrome de Lennox Gastaut, afortunadamente actualmente controlada.

\section{BIBLIOGRAFIA}

1.Hagberg BA. Rett síndrome; clinical peculiarities diagnostic approach and posible cause. Pediatr Neurol 1989; 5: 75-83.

2.Hagberg BA, Witt-Engerstrom I. Rett syndrome: a soggested staging system for describing impaiment profile with increasing age towards adolescence. Am Med Genet 1986; 24: 47-59.

3.Lugaresi E, Cirignotta F, Montana P. Breathing in the Rett Syndrome. Brain Dev 1985; 7: 329-333.

4.Hagberg BA, Aicardi J. Dias K, Ramos O.: A progressive syndrome of autism, dementia, ataxia and loss of urposeful hand use in girls: Rett's syndrome: Report of 35 cases. Ann Neurol 1983; 14: 471-479.

5. Hagberg BA, Rett's syndrome: prevalence and impact on progressive severe mental retardation in girls. Acta Paediatr Scand 1985; 74: 405-408.

6. Dorries A, Spohr Hl, Kunze J. Angelman ("happy puppet") syndrome seven new cases documented by cerebral computed tomography: Review of the literature. Eur. J Pediatr 1988; 148:270.

7.Hagbne I, Witt-Engerstr: om I. Hagberg BA. EEG development in Rett syndrome. A study of cases. EEG Clin. Neurophysiol 1989; 72: 1-6.

8. Suzuli H, Matsuzaka T, Hirayama Y et al.: Rett's syndrome. Proggresive of symptoms from infancy to childhood. J Child Nerol 1986; 1: 137-141.

9.Glaze DG, Frost JD, Zoghbi HY, Percy AK. Rett's syndrome Correlation of electroencephalographic characteristic with clinical staging. Neurol 1987; 44: 10351038 .

10.Killian W. On the genetics of Rett syndrome: analysis of family and pedigree data Am J Med Genet 1986; 24(suppl 1): 369-376.

11. Percy A, Gilberg CH, Hagberg BA, Witt-Engerstr, OL. Rett syndrome and autistic disorders. Neurologic clinica 1990; 8: 659-676.

12. Robb SA, Pohl KRE, Baraitser Metal "The happy puppet"syndrome of Angelman. Review of clinical features. Arch Dis child 1989; 64:83.

14. Zoghbi H. Genetics aspects of Rett syndrome.J Child Neurol 1988; 3; 76-78. 